ENSLAPP-A-655/97

CCNY-HEP 97/6

La Plata Th-16/97

\title{
Duality between Topologically Massive and Self-Dual models
}

\author{
J.C. Le Guillou ${ }^{a *}$, E. F. Moreno ${ }^{b \dagger}$, C. Núñez ${ }^{c}$ \\ and \\ F.A. Schaposnik ${ }^{c \ddagger}$ \\ ${ }^{a}$ Laboratoire de Physique Théorique ENSLAPP $\S$ \\ LAPP, B.P. 110, F-74941 Annecy-le-Vieux Cedex, France \\ ${ }^{b}$ Physics Department, City College of the City University of New York \\ New York NY 10031, USA \\ Physics Department, Baruch College, The City University of New York \\ New York NY 10010, USA \\ ${ }^{c}$ Departamento de Física, Universidad Nacional de La Plata \\ C.C. 67, 1900 La Plata, Argentina
}

\begin{abstract}
We show that, with the help of a general BRST symmetry, different theories in 3 dimensions can be connected through a fundamental topological field theory related to the classical limit of the ChernSimons model.
\end{abstract}

*Also at Université de Savoie and at Institut Universitaire de France

†Supported by CUNY Collaborative Incentive Grant 991999

${ }^{\ddagger}$ Investigador CICBA, Argentina

§URA 1436 du CNRS associée à l'Ecole Normale Supérieure de Lyon et à l'Université de Savoie 
Different descriptions of massive self-dual vector fields in three dimensions have been shown to exists both in the Abelian and non-Abelian cases [1]]- [4]. The action is either linear in derivatives (the self-dual model) or quadratic with a topological mass term [5]. The connection thus established between different models was recently exploited to analyze relevant supersymmetric models [4] and also to derive bosonization rules for three dimensional fermionic systems [6]. A phase space analysis of the connection in the abelian case can be found in [7].

Trying to overcome some complications that arise in establishing the connection in the non-Abelian case [4] a "large" BRST invariance was exploited in ref. 80 in connection with non-Abelian bosonization in $d=3$ dimensions. It is the purpose of this work to use this BRST invariance to show that the different models studied in [1]-[4] can all be described by a unique partition function related to the classical limit of the Chern-Simons theory partition function.

Let us start by considering the following (Euclidean) path-integral for a gauge field $b_{\mu}$ taking values in the Lie algebra of some group $G$

$$
Z=\sum_{\alpha} \int \mathcal{D} b_{\mu} \delta\left[b_{\mu}-\tilde{b}_{\mu}^{(\alpha)}\right] \exp \left(\frac{\kappa}{2 \pi} S_{C S}[b]\right)
$$

Here $S_{C S}[b]$ is the Chern-Simons action,

$$
S_{C S}[b]=i \varepsilon_{\mu \nu \lambda} \operatorname{tr} \int_{M} d^{3} x\left(F_{\mu \nu}[b] b_{\lambda}-\frac{2}{3} b_{\mu} b_{\nu} b_{\lambda}\right),
$$

$\tilde{b}_{\mu}^{(\alpha)}$ is a complete set of gauge equivalence classes of flat connections and $M$ an oriented three manifold. Note that the constraint on the path-integration domain imposed by the delta functional in eq.(1) corresponds to saturate the path-integral with the solution of the classical equations of motion for the Chern-Simons action,

$$
\varepsilon_{\mu \nu \lambda} F_{\mu \nu}[b]=0 .
$$

In this sense, we could think of $Z$ as the partition function for a Chern-Simons theory restricted to the classical sector. With this restriction, the CS theory which is a Schwartz type topological theory becomes a Witten type topological theory [9]. Indeed, if one considers the most general transformation for $b_{\mu}$,

$$
b_{\mu} \rightarrow b_{\mu}+\epsilon_{\mu}
$$


which leaves invariant (Witten-type) topological Yang-Mills theory, the CS action changes as

$$
\delta_{\epsilon_{\mu}} S_{C S}=2 i \varepsilon_{\mu \nu \alpha} \int d^{3} x \epsilon_{\mu} F_{\nu \alpha}[b]
$$

But, when the $\delta$-function constraint is included as in (1), this non-invariant term vanishes. Then, the model defined through partition function (11) has a "large" topological invariance as it occurs in Witten type theories and, associated with it, it posses, as we shall see, a "large" BRST invariance. Now, let us note that integrating the delta function in (ID) one just gets

$$
Z=\sum_{\alpha} \exp \left(\frac{\kappa}{2 \pi} S_{C S}\left[\tilde{b}^{(\alpha)}\right]\right)
$$

We then see that the partition function (11) picks contributions solely from the classical sector of the CS theory since only the phase of the CS invariant of the flat connection $b^{(\alpha)}, S_{C S}\left[b^{(\alpha)}\right]$, appears in (6). This should be contrasted with the "complete" Chern-Simons theory, i.e. that with a partition function of the form

$$
Z_{C S}=\int \mathcal{D} b_{\mu} \exp \left(\frac{\kappa}{2 \pi} S_{C S}[b]\right)
$$

Now, due to eq.(6), it is evident that one can connect the theory defined by (11) with the weak coupling limit of the complete CS theory since

$$
\lim _{\kappa \rightarrow \infty} \int \mathcal{D} b_{\mu} \exp \left(\frac{\kappa}{2 \pi} S_{C S}[b]\right)=\lim _{\kappa \rightarrow \infty} \sum_{\alpha} \exp \left(\frac{\kappa}{2 \pi} S_{C S}\left[\tilde{b}^{(\alpha)}\right]\right)
$$

or, more compactly

$$
\lim _{\kappa \rightarrow \infty} Z_{C S}=\lim _{\kappa \rightarrow \infty} Z
$$

That is, the limit of $\kappa \rightarrow \infty$ of our model coincides with the same limit of the (complete) CS model.

According to the manifold, $Z_{C S}$ can be evaluated using different approaches [10-11]. In particular, for a manifold $M=S^{2} \times S^{1}$, one has $Z_{C S}\left[S^{2} \times S^{1}, G\right]=1$ for any $G$ and any $k$, a result which can be considered as a normalization definition for the partition function of CS theories in manifolds of the form $X \times S^{1}$. For $M=S^{3}$ and $G=S U(2) Z_{C S}$ can also be evaluated, leading to

$$
Z_{C S}\left[S^{3}, S U(2)\right]=\sqrt{\frac{2}{k+2}} \sin \left(\frac{\pi}{k+2}\right)
$$


which behaves as $Z \sim k^{-3 / 2}$ for large k. Finally, under some circumstances (in particular, when there are no ghost and auxiliary fields zero modes) the partition function for the Chern-Simons theory can be studied in the weak coupling limit. We thus see that according to the manifold and the group, one can compute the $k \rightarrow \infty$ limit of the CS partition function which can be identified with our starting partition function (1).

In order to make apparent the BRST invariance referred above, let us concentrate on an element of the equivalent class of flat gauge connections (i.e. on a given term in the sum defined in eq.(1)) and write the delta functional of eq. (11) in the form [8]

$$
\delta\left[b_{\mu}-g^{-1} \partial_{\mu} g\right]=\left|\operatorname{det}\left(2 \varepsilon_{\mu \nu \alpha} D_{\nu}[b]\right)\right| \delta\left[\varepsilon_{\mu \nu \lambda} F_{\mu \nu}[b]\right]
$$

where $D_{\mu}[b]=\partial_{\mu}+\left[b_{\mu},\right]$. With this, the partition function (11) can be rewritten as

$$
Z=\int \mathcal{D} b_{\mu} \mathcal{D} A_{\mu} \mathcal{D} \bar{c}_{\mu} \mathcal{D} c_{\mu} \exp \left(-S_{e f f}[b, A, \bar{c}, c]\right)
$$

where

$$
S_{e f f}[b, A, \bar{c}, c]=-\frac{1}{2 \pi} S_{C S}[b]-\frac{i}{\pi} \varepsilon_{\mu \nu \alpha} t r \int d^{3} x\left(A_{\mu} F_{\nu \alpha}[b]-2 \bar{c}_{\mu} D_{\nu}[b] c_{\alpha}\right) .
$$

Here $A_{\mu}$ is a one-form Lagrange multiplier introduced to represent the delta functional and $\bar{c}_{\mu}$ and $c_{\mu}$ are one-form ghost fields introduced to exponentiate the determinant in (11).(We have omitted a group volume factor in (12).) The partition function (12) can be rewritten as

$$
Z=\int \mathcal{D} b_{\mu} \mathcal{D} A_{\mu} \mathcal{D} \bar{c}_{\mu} \mathcal{D} c_{\mu} \mathcal{D} d_{\mu} \mathcal{D} l \mathcal{D} \bar{\xi} \exp \left(-\tilde{S}_{e f f}[b, A, \bar{c}, c, d, l, \bar{\xi}]\right)
$$

where

$$
\begin{aligned}
& \tilde{S}_{e f f}[b, A, \bar{c}, c, d, l, \bar{\xi}]=-\frac{1}{2 \pi} S_{C S}[b-d]+\frac{i}{\pi} \operatorname{tr} \int d^{3} x\left(-l d_{\mu} d_{\mu}+2 \bar{\xi} c_{\mu} d_{\mu}\right) \\
& -\frac{i}{\pi} \varepsilon_{\mu \nu \alpha} \operatorname{tr} \int d^{3} x\left[\left(A_{\mu}+2 d_{\mu}\right) F_{\nu \alpha}[b]-2 \bar{c}_{\mu} D_{\nu}[b] c_{\alpha}\right]
\end{aligned}
$$

Here $d_{\mu}$ is a one form auxiliary field taking values in the Lie algebra, $l$ a scalar and $\bar{\chi}$ an antighost field. Integrating out $l$ constrains $d_{\mu}$ to be zero so 
that (14) is trivially equivalent to (12). One can see that $\tilde{S}_{\text {eff }}$ possesses an (off-shell nilpotent) BRST symmetry with transformations defined as

$$
\begin{array}{cc}
\delta b_{\mu}=c_{\mu} & \delta c_{\mu}=0 \\
\delta \bar{c}_{\mu}=A_{\mu}+2 d_{\mu} & \delta A_{\mu}=-2 c_{\mu} \quad \delta d_{\mu}=c_{\mu} \\
\delta \bar{\xi}=l & \delta l=0
\end{array}
$$

According to (16), $\tilde{S}_{\text {eff }}$ can be rewritten in the form

$$
\tilde{S}_{e f f}=-\frac{1}{2 \pi} S_{C S}[b-d]-\frac{i}{\pi} \operatorname{tr} \int d^{3} x \delta\left(\bar{c}_{\mu}{ }^{*} F_{\mu}[b]+\bar{\xi} d_{\mu} d_{\mu}\right)
$$

and we have defined ${ }^{*} F_{\mu}[b]=\varepsilon_{\mu \nu \alpha} F_{\nu \alpha}[b]$. In view of the BRST invariance we can add BRST exact forms to $\tilde{S}_{e f f}$, without changing the physics of the model. If we choose a functional $\mathcal{G}=i\left(g^{2} / \pi\right) \int d^{3} x \bar{c}_{\mu} A_{\mu}$ with $g$ an arbitrary constant carrying units of mass, $\left[g^{2}\right]=m$ and add $\delta \mathcal{G}$ to the effective action one generates a mass term for the $A_{\mu}$ field so that, after integrating out $l$ giving $d_{\mu}=0$, one finally ends with

$$
\begin{aligned}
\tilde{S}_{e f f}[b, A, \bar{c}, c]= & -\frac{1}{2 \pi} S_{C S}[b]-\frac{i}{\pi} \operatorname{tr} \int d^{3} x A_{\mu}{ }^{*} F_{\mu}[b]+\frac{i g^{2}}{\pi} \operatorname{tr} \int d^{3} x A_{\mu} A_{\mu}+ \\
& \frac{2 i}{\pi} \operatorname{tr} \int d^{3} x \bar{c}_{\mu}\left({ }^{*} D_{\mu \alpha}[b]-g^{2} \delta_{\mu \alpha}\right) c_{\alpha}
\end{aligned}
$$

We thus see that $\widetilde{S}_{\text {eff }}$ can be interpreted as the quantum version of the "master" action introduced, in the Abelian case, in refs. [2] in order to establish the equivalence between the massive self-dual theory and the topologically massive gauge theory [1]. In the abelian case one can easily see this connection starting from the master action and integrating out either $A_{\mu}$ or $b_{\mu}$ [6]. Now, in the non-Abelian case, due to non-quadratic terms appearing in the CS action, this exact integration is not possible so that the equivalence between the massive non-Abelian self-dual model and Yang-Mills-Chern-Simons (or topologically massive) theory cannot be proven in such a way.

More specifically, starting from

$$
Z=\int \mathcal{D} A \mathcal{D} b \mathcal{D} \bar{c} \mathcal{D} c \exp \left(-\tilde{S}_{e f f}[b, A, \bar{c}, c]\right)
$$


one can easily perform the $A_{\mu}$ integration so that $Z$ takes the form

$$
\begin{aligned}
Z= & \int \mathcal{D} b \mathcal{D} \bar{c} \mathcal{D} c \exp \left(\frac{1}{2 \pi} S_{C S}[b]+\frac{i}{2 \pi g^{2}} \operatorname{tr} \int d^{3} x F_{\mu \nu}^{2}[b]\right) \times \\
& \exp \left(-\frac{2 i}{\pi} \operatorname{tr} \int d^{3} x \bar{c}_{\mu}\left({ }^{*} D_{\mu \alpha}[b]-g^{2} \delta_{\mu \alpha}\right) c_{\alpha}\right) .
\end{aligned}
$$

We thus see that, at least in the strong-coupling regime $\left(g^{2} \rightarrow \infty\right)$, where the ghost integration trivially decouples, $Z$ corresponds to the partition function of topologically massive gauge theory: $\lim _{g^{2} \rightarrow \infty} Z=Z_{\text {top }}$.

Now, as stated above, interchanging the order of integration (first integrating over $b_{\mu}$ ) does not lead to a simple theory for the $A$ field (as one does in the Abelian case). However, if exploiting BRST invariance we add to $\tilde{S}_{\text {eff }}$ a BRST exact form $\delta \mathcal{H}$, thus defining :

$$
\hat{S}_{e f f}=\tilde{S}_{e f f}-\frac{2 i}{\pi} \epsilon_{\mu \nu \alpha} \int d^{3} x \delta\left\{\bar{c}_{\mu}\left(\left[b_{\nu}, A_{\alpha}+2 d_{\alpha}\right]-\left(b_{\nu}-d_{\nu}\right)\left(A_{\alpha}+2 d_{\alpha}\right)\right)\right\}
$$

then, after integration over auxiliary fields, non-quadratic terms accommodate in the form first written in [3] so that the $b_{\mu}$ integration can be trivially factored out. Indeed, $\hat{S}_{\text {eff }}$ takes the form

$$
\begin{aligned}
& \hat{S}_{e f f}=-\frac{1}{2 \pi} S_{C S}[b]+\frac{i g^{2}}{\pi} \operatorname{tr} \int d^{3} x A_{\mu} A_{\mu}-\frac{i}{\pi} \operatorname{tr} \int d^{3} x A_{\mu}^{*} F_{\mu}[b] \\
& -\frac{2 i}{\pi}\left(\varepsilon_{\mu \nu \alpha} \operatorname{tr} \int d^{3} x A_{\mu} A_{\nu} b_{\alpha}-\bar{c}_{\mu}\left({ }^{*} D_{\mu \alpha}[b+A]-g^{2} \delta_{\mu \alpha}\right) c_{\alpha}\right) .
\end{aligned}
$$

After performing the shift $b_{\mu} \rightarrow b_{\mu}-A_{\mu}$, the integration over $b_{\mu}$ and the ghost fields is factored out leading to the partition function of the Self-Dual model,

$$
Z=\int \mathcal{D} A_{\mu} \exp \left(-S_{S D}[A]\right)
$$

with

$$
S_{S D}[A]=\frac{1}{2 \pi} S_{C S}[A]+\frac{i g^{2}}{\pi} \operatorname{tr} \int d^{3} x A_{\mu} A_{\mu},
$$

result which was not obtained in ref. [3].

We have then established a new series of equivalencies which is summarized in Figure 1. The original constrained Chern-Simons model becomes, after addition of a BRST exact form $\delta \mathcal{G}$ an effective model which, after 
integration over the Lagrange multiplier $A_{\mu}$, corresponds to a class of topologically massive model. In fact, in the $g^{2} \rightarrow \infty$ limit it coincides with the Yang-Mills-Chern-Simons theory originally introduced to topologically generate a mass for the gauge field [5]. If one still adds an appropriate BRST variation the "integration over the field $b_{\mu}$ " can be easily performed resulting in the partition function for a self-dual model.

It is important at this point to analyze the issue of symmetries in the different models we have connected. We started with a constrained CS model endowed with gauge symmetry. Indeed, the theory defined by partition function (11) shows an invariance under the gauge transformation

$$
\begin{gathered}
b_{\mu} \rightarrow h^{-1} b_{\mu} h+h^{-1} \partial_{\mu} h \\
g \rightarrow h g .
\end{gathered}
$$

This symmetry is maintained after introduction of the Lagrange multiplier field $A_{\mu}$ and ghosts provided their transformation laws are of the form

$$
A_{\mu} \rightarrow h^{-1} A_{\mu} h, \quad c_{\mu} \rightarrow h^{-1} c_{\mu} h, \quad \bar{c}_{\mu} \rightarrow h^{-1} \bar{c}_{\mu} h
$$

Now, after addition of the BRST exact form to pass from $\tilde{S}_{\text {eff }}$ to $\hat{S}_{\text {eff }}$ this invariance is spoiled. It is then no surprise that we arrive to a self-dual model in which the presence of the CS action makes evident that transformation (27), ensuring that $A_{\mu}$ transforms covariantly and not as a gauge field, does not leave invariant the action. Remarkably, one can overcome this problem by introducing a supplementary vector field $V_{\mu}$ precisely as advocated in ref. [3].

Indeed, let us consider the following action, modification of $\tilde{S}_{\text {eff }}$ by addition of the field $V_{\mu}$ taking values in the Lie algebra of $G$,

$$
S_{e f f}=\tilde{S}_{e f f}[b, A, \bar{c}, c, d, l, \bar{\xi}]+S[V-d]+\delta \mathcal{K} .
$$

Here $S[V]$ is the action giving dynamics to the $V_{\mu}$ field and $\mathcal{K}$ will be chosen so as to establish connections, analogous to those discussed above, but now in the presence of the vector field $V_{\mu}$. Consider the partition function

$$
Z=\int \mathcal{D} \Phi \exp \left(-S_{e f f}\right)
$$


where $\Phi$ represent the complete set of fields now including $V_{\mu}$. We impose to $V_{\mu}$ the BRST transformation law

$$
\delta V_{\mu}=c_{\mu}
$$

and supplement symmetry transformations (27) with

$$
V_{\mu} \rightarrow g^{-1} V_{\mu} g+g^{-1} \partial_{\mu} g
$$

Let us consider a family of functionals $\mathcal{K}$, depending on a parameter $\lambda$, defined as

$\mathcal{K}[V, b, A, \bar{c} ; \lambda]=-\frac{i}{\pi} \varepsilon_{\mu \nu \alpha} \int d^{3} x\left(\bar{c}_{\mu}\left[b_{\nu}-V_{\nu}, A_{\alpha}\right]+\lambda \bar{c}_{\mu}\left(A_{\nu}+2 d_{\nu}\right)\left(A_{\alpha}+2 d_{\alpha}\right)\right)$

One can easily see that the resulting effective action is invariant (after integration over auxiliary fields) under gauge transformations (27), (31). Indeed, $S_{\text {eff }}$ reads, after integration on auxiliary fields, :

$$
\begin{aligned}
& S_{e f f}=-\frac{1}{2 \pi} S_{C S}[b]+\frac{i g^{2}}{\pi} \operatorname{tr} \int d^{3} x A_{\mu} A_{\mu}-\frac{i}{\pi} \operatorname{tr} \int d^{3} x A_{\mu}^{*} F_{\mu}[b]+S[V] \\
& -\frac{2 i}{\pi}\left(\varepsilon_{\mu \nu \alpha} \operatorname{tr} \int d^{3} x A_{\mu} A_{\nu}\left(b_{\alpha}-V_{\alpha}+\frac{\lambda}{2} A_{\alpha}\right)-\bar{c}_{\mu}\left({ }^{*} D_{\mu \alpha}[V]-g^{2} \delta_{\mu \alpha}\right) c_{\alpha}\right)
\end{aligned}
$$

which corresponds for $\lambda=0$ to the quantum version of the action eq. (11) of ref. $([3])$.

Now, with the choice $\lambda=0, S_{\text {eff }}$ becomes quadratic in $A_{\mu}$ and one can perform the path-integral thus obtaining

$$
Z=\int \mathcal{D} b_{\mu} \mathcal{D} V_{\mu} \exp (-S[b, V])
$$

with

$$
\begin{aligned}
S[b, V]= & -\frac{1}{2 \pi} S_{C S}[b]+S[V]+\log \operatorname{det}\left(g^{2} \delta_{\mu \alpha}-{ }^{*} D_{\mu \alpha}[V]\right) \\
& -\frac{i}{4 \pi} \operatorname{tr} \int d^{3} x^{*} F_{\mu}[b]\left[g^{2} \delta_{\mu \alpha}-2 \varepsilon_{\mu \nu \alpha}\left(b_{\nu}-V_{\nu}\right)\right]^{-1}{ }^{*} F_{\alpha}[b] \\
& +\frac{1}{2} \log \operatorname{det}\left[g^{2} \delta_{\mu \alpha}-2 \varepsilon_{\mu \nu \alpha}\left(b_{\nu}-V_{\nu}\right)\right]
\end{aligned}
$$

This expression corresponds to the quantum version of the action discussed in ref.([3]) (see eq.(13) of that paper). 
If one instead tries to first integrate out $b_{\mu}$, cubic terms prevent explicit integration. However, one can perform a change of variables that factors out trivially this integration leaving the exact partition function for the $A_{\mu}$ and $V_{\mu}$ fields. Indeed, if we define $b_{\mu}^{\prime}=b_{\mu}+A_{\mu}$ the effective action reads, in terms of the new variable $b^{\prime}$ as

$$
S_{e f f}=-\frac{1}{2 \pi} S_{C S}\left[b^{\prime}\right]+\hat{S}[A, V]
$$

where

$$
\begin{aligned}
& \hat{S}[A, V]=\frac{i}{\pi} \varepsilon_{\mu \nu \alpha} \operatorname{tr} \int d^{3} x\left(A_{\mu} D_{\nu}[V] A_{\alpha}+\left(\frac{2}{3}-\lambda\right) A_{\mu} A_{\nu} A_{\alpha}\right) \\
& +\frac{i g^{2}}{\pi} \operatorname{tr} \int d^{3} x A_{\mu} A_{\mu}+S[V]+\log \operatorname{det}\left(g^{2} \delta_{\mu \alpha}-{ }^{*} D_{\mu \alpha}[V]\right)
\end{aligned}
$$

At the partition function level, the $b_{\mu}^{\prime}$ integral trivially factors out so that $\hat{S}[A, V])$ determines the quantum theory which, for $\lambda=1 / 3$, corresponds to the first order action discussed in [3] (see eq.(9) in this reference). Finally, for $\lambda=2 / 3$ we obtain an action $S[A, V]$ which makes contact with the starting action of eq. (1) in that paper, describing the dynamics of a massive self-dual non-Abelian gauge field.

We have thus shown both the quantum version of the dualization of the ref. [3], dualization which was only achieve in ref. [3] using in fact a modified version of its eq. (1), and the existence of a quantum dualization using strictly this eq. (1) at the classical level.

This extends the picture we established when $V_{\mu}$ was absent connecting self-dual models with topologically massive models and summarized in Figure 1. By incorporating the $V_{\mu}$ field, we have extended these connections so as to include models which were discussed in the context of Supergravity in ref. [G]. The new connections are now displayed in Figure 2.

Up to this point our analysis was presented at the level of the partition functions of the different models. We shall end this work by briefly explaining how we can incorporate external sources to promote these connections to the level of generating functionals of Green's functions. In particular, we shall be able to establish that the Green's functions for $A_{\mu}$ in the self-dual model with partition function (23) are those for $\varepsilon_{\mu \nu \alpha} F_{\nu \alpha}[b]$ in the topologically massive model with partition function (20), showing thus the following connection :

$$
A_{\mu} \rightarrow \varepsilon_{\mu \nu \alpha} F_{\nu \alpha}[b] \text {. }
$$


To this end, let us consider the following path-integral :

$$
Z[s]=\int \mathcal{D} b_{\mu} \delta\left[b_{\mu}-\tilde{b}_{\mu}(s)\right] \exp \left(\frac{1}{2 \pi} S_{C S}[b]\right)
$$

where $\tilde{b}_{\mu}(s)$ is defined through

$$
\varepsilon_{\mu \nu \alpha} F_{\nu \alpha}[\tilde{b}(s)]=s_{\mu} .
$$

After exponentiating the delta functionals by introducing as in the sourceless case a Lagrange multiplier $A_{\mu}$, introducing ghosts for the Faddeev-Popov determinant and auxiliary fields, and using BRST transformations to write $A_{\mu} s_{\mu}=\left(\delta \bar{c}_{\mu}\right) s_{\mu}$, one ends with the analogous of eqs. (18, 19)

$$
Z[s]=\int \mathcal{D} b_{\mu} \mathcal{D} A_{\mu} \mathcal{D} \bar{c}_{\mu} \mathcal{D} c_{\mu} \exp \left(-\tilde{S}_{e f f}[b, A, \bar{c}, c ; s]\right)
$$

with

$$
\begin{aligned}
\tilde{S}_{e f f}[b, A, \bar{c}, c ; s]= & -\frac{1}{2 \pi} S_{C S}[b]-\frac{i}{\pi} \operatorname{tr} \int d^{3} x A_{\mu}{ }^{*} F_{\mu}[b]+\frac{i g^{2}}{\pi} \operatorname{tr} \int d^{3} x A_{\mu} A_{\mu}+ \\
& \frac{2 i}{\pi} \operatorname{tr} \int d^{3} x \bar{c}_{\mu}\left({ }^{*} D_{\mu \alpha}[b]-g^{2} \delta_{\mu \alpha}\right) c_{\alpha}+\frac{i}{\pi} \operatorname{tr} \int d^{3} x s_{\mu} A_{\mu}(42)
\end{aligned}
$$

As in the sourceless case, if we "integrate (41) over $b_{\mu}$ ", i.e. adding (before integration over the auxiliary fields) the BRST exact form $\delta \mathcal{H}$ as in (21) and performing the shift $b \rightarrow b-A$, we end with the generating functional for the self-dual model :

$$
Z[s]=\int \mathcal{D} A_{\mu} \exp \left(-S_{S D}[A]+\frac{i}{\pi} \operatorname{tr} \int d^{3} x s_{\mu} A_{\mu}\right)
$$

If instead we integrate (41) over $A_{\mu}$ we end with a generating functional for the topologically massive model :

$$
\begin{aligned}
& Z[s]=\int \mathcal{D} b \mathcal{D} \bar{c} \mathcal{D} c \exp \left(\frac{1}{2 \pi} S_{C S}[b]+\frac{i}{2 \pi g^{2}} \operatorname{tr} \int d^{3} x F_{\mu \nu}^{2}[b]-\right. \\
& \left.\frac{2 i}{\pi} \operatorname{tr} \int d^{3} x \bar{c}_{\mu}\left({ }^{*} D_{\mu \alpha}[b]-g^{2} \delta_{\mu \alpha}\right) c_{\alpha}-\frac{i}{2 \pi g^{2}} \operatorname{tr} \int d^{3} x s_{\mu}\left({ }^{*} F_{\mu}[b]+\frac{s_{\mu}}{2}\right)\right)
\end{aligned}
$$

The equivalence between (43) and (44) implies by differentiation the connection (38) up to a coefficient. The same procedure can be followed in order 
to add sources to the model defined by the partition function (29) which includes the supplementary field $V_{\mu}$. We shall not repeat the steps here.

In conclusion: different descriptions of massive self-dual vector field theory in three dimensions can be linked together with the aid of a general BRST symmetry. Moreover, all of these models are descendent of a primal topological field theory, related to the classical limit of the CS theory. The introduction of external sources allows us to find the precise correspondence between the basic fields in the different theories.

Acknowledgments: F.A.S. and C.N. are partially supported by Fundacion Antorchas, Argentina and a Commission of the European Communities contract No:C11*-CT93-0315. E.M. is partially supported by CUNY Collaborative Incentive Grant 991999.

\section{References}

[1] P.K. Townsend, K. Pilch and P. van Nieuwenhuizen, Phys. Lett. B136 (1984) 38; B137 (1984) 443.

[2] S. Deser and R. Jackiw, Phys.Lett. B139 (1984) 371.

[3] A. Karlhede, U. Lindström, M. Roček and P. van Nieuwenhuizen, Phys. Lett. 186B (1987) 96.

[4] A. Karlhede, U. Lindström, M. Roček and P. van Nieuwenhuizen, Class. Quantum Grav. 4 (1987) 549.

[5] R. Jackiw and S. Templeton, Phys. Rev. D23 (1981) 2291; S. Deser, R. Jackiw and S. Templeton, Phys. Rev. Lett. 48 (1982) 975; Ann. of Phys. (N.Y.) 140 (1982) 372.

[6] E. Fradkin and F.A. Schaposnik, Phys. Lett. B338 (1994) 253; N. Bralić, E. Fradkin, M.V. Manías and F.A. Schaposnik, Nucl. Phys. 446 (1995) 144.

[7] R. Banerjee, H.J. Rothe and K.D. Rothe, Phys. Rev. D52 (1995) 3750. 
[8] J.C. Le Guillou, E. Moreno, C. Núñez and F.A.Schaposnik, Nucl. Phys. B484 (1997) 682; On three dimensional bosonization, hep-th 9703048, to be published in Phys.Lett. B.

[9] D. Birmingham, M. Blau, M. Rakowski and G. Thompson, Phys. Rep. 209 (1991) 129.

[10] E. Witten Commun. Math. Phys 121 (1989) 351.

[11] J.M. Isidro, J.M.F. Labastida and A.V. Ramallo, Nucl. Phys. 398B (1993) 187. 


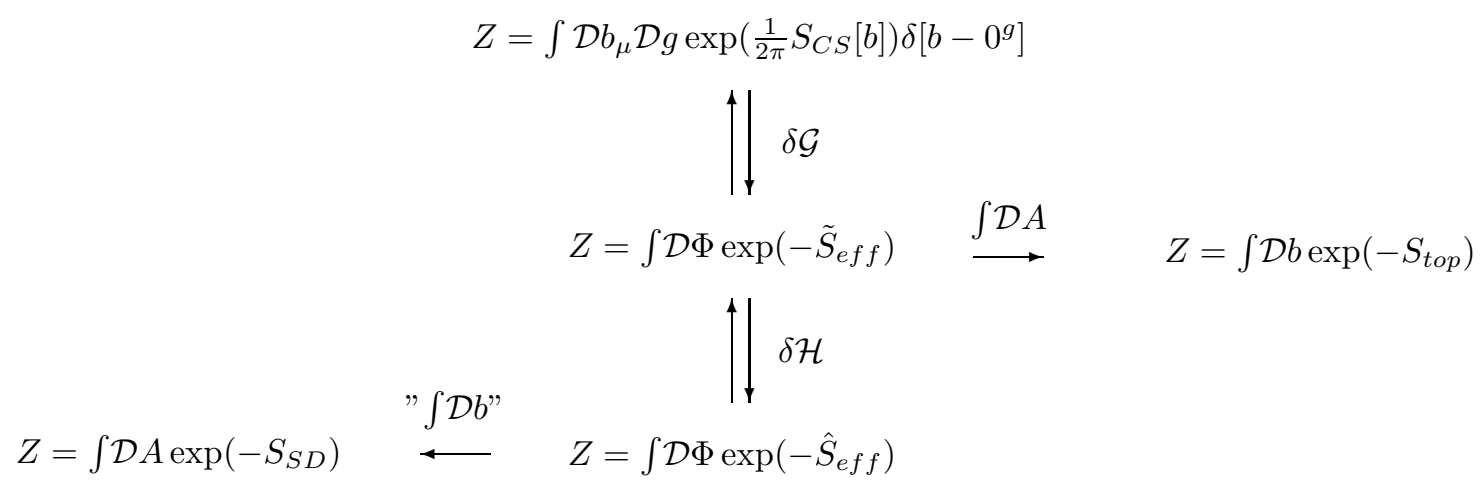

Self-Dual Models

Topologically Massive Models

Figure 1

Connections between different models.

$Z=\int \mathcal{D} \Phi \exp \left(-\tilde{S}_{\text {eff }}-S[V]\right)$
$\Uparrow \mid \delta \mathcal{K}(\lambda)$
$Z=\int \mathcal{D} A \mathcal{D} V \exp (-S[A, V]) \stackrel{\frac{2}{3}, " \int \mathcal{D} b "}{\longleftarrow} Z=\int \mathcal{D} \Phi \exp \left(-S_{\text {eff }}\right) \stackrel{\lambda=0, \int \mathcal{D} A}{\longrightarrow} Z=\int \mathcal{D} b \mathcal{D} V \exp (-S[b, V])$

Self-Dual Models

Topologically Massive Models

Figure 2

New connections when the vector field $V_{\mu}$ is considered. 